\title{
An Energy-Efficient Strategy for VoD Content Replication in Integrated Metro/Access Networks
}

\author{
M. Savi, G. Verticale, M. Tornatore and A. Pattavina
}

\begin{abstract}
The Video-on-Demand (VoD) service is becoming one the most popular Internet services. Due to its bandwidthconsuming nature and thanks to the efforts made by telecom operators to provide the users with higher access rates, an huge amount of VoD traffic is expected to flood the core segment of the network, potentially leading to congestion and Quality of Experience degradation issues. A growing body of research is studying how to alleviate such traffic explosion. One of the most promising solutions consists in migrating the content in the metro and access segments of the network by deploying a system of Metro Servers (MSes) for VoD delivery. This way, the VoD traffic is kept as local as possible and the core network is trafficoffloaded. This paper investigates one of the most prominent risks by adopting this solution: in fact, if not carefully managed, a massive deployment of MSes in the network can lead to a significant increase of the overall energy consumption of the network. We first identify a consistent power model for the MSes and the network equipment in an integrated metro/access architecture scenario, then we define a strategy to switch on and off the network interfaces and the MSes depending on the VoD traffic load variation during the day. We show by means of simulations how our strategy always strikes the best trade-off between the energy consumption for content transport through the network and the energy consumption for processing and storage in the MSes.
\end{abstract}

Keywords - Energy efficiency, Access network, Metro network, Video-on-Demand, Content Delivery.

\section{INTRODUCCIÓN}

$\mathrm{I}^{\mathrm{N}}$ NTERNET fijo y el trafico móvil esta en constante aumento [1]. Hay varias causas detrás de este crecimiento sostenido del trafico. Entre ellos, esta la adopción de servicios de video streaming de banda ancha, como Video bajo Demanda (VoD), que aumenta año tras año debido al incremento en el compromiso de los usuarios con los servicios multimedia innovadores. En pocos años de los medios streaming y especialmente el trafico de video será dominante en la infraestructura de red. Los operadores Telecom están realizando grandes esfuerzos para proporcionar a los usuarios con mayores tasas de acceso mediante el desarrollo innovativo de tecnologías fijas y móvil de accesos de red, como fibra hasta la casa (FTTH), fibra hasta el edificio (FTTB) o Long Term Evolution (LTE).

Redes de Telecomunicaciones pueden ser mas o menos dividida en tres diferentes e interconectados segmentos: segmento de acceso, segmento de metro y segmento core. En el actual escenario, los contenidos de video son usualmente almacenados en un servidor centralizado y/o los centros de datos son colocados en la red core. La explosión de videotrafico contribuiría significativamente en la inundación de la

M. Savi, Politecnico di Milano, Italy, marco.savi@polimi.it

G. Verticale, Politecnico di Milano, Italy, giacomo.verticale@polimi.it

M. Tornatore, Politecnico di Milano, Italy, massimo.tornatore@polimi.it

A. Pattavina, Politecnico di Milano, Italy, achille.pattavina@polimi.it red core, que podría convertirse en el nuevo cuello de botella en la infraestructura general, comprometiendo severamente la calidad de todos los servicios de Internet.

Una posible solución para evitar dicho escenario es emigrar los servidores de contenido de video mas cerca de los usuarios, posiblemente en el segmento metro y/o en el segmento de acceso. Con el despliegue de un sistema de servidores de video distribuidos (que puede ser llamado Metro Servers (MSes)) en estos segmentos de la red, una gran cantidad de trafico VoD se puede mantener de forma local. La red core se beneficiara de esta descarga y los usuarios experimentaran una mejor calidad de experiencia (QoE), ya que la latencia de la entrega de contenido disminuirá considerablemente.

A pesar de la ventajas en términos de descarga y latencia son evidentes, el uso de servidores de video en el área metro/acceso deben ser estudiados cuidadosamente y monitoreo para evitar in incremento indiscriminado en la energía de consumo en estos segmentos de red. En algunos estudios existentes [2][3][4][5], ya ha sido demostrado que el despliegue de los servidores $\mathrm{VoD}$ distribuidos con respecto a una solución centralizada conduce a dos efectos contrastantes. Por un lado, el consumo total de energía para el transporte p.ej., la energía que se necesita para entregar un video streaming a través de la red, es reducida, ya que algunos de los contenidos de video son entregados a los usuarios desde una ubicación mas cercana. Por otro lado, la energía total necesaria para el almacenamiento y procesamiento de datos (es decir, el consumo de energía almacenado en la caché) de los contenidos de video incrementa debido a la gran cantidad de servidores de video que deben ser colocados y encendidos en la red metro/acceso. Por lo tanto, de acuerdo a las condiciones de trafico, podría ser mas eficiente enérgicamente cambiar a MSes on o off, y, según nuestro conocimiento, no se ha realizado ningún estudio para investigar la forma como gestionar el estado de energía del MSes en presencia de solicitudes de trafico que cambia dinámicamente.

En este trabajo, comenzado desde la investigación acerca del consumo de energía en la entrega de VoD en presencia de MSes, propondremos y evaluaremos nuevas estrategias para coordinar la conmutación on/off de las interfaces de red y MSes dependiendo de las condiciones de carga de trafico durante el día. Estas estrategias permitirán al operador VoD ahorrar gran cantidad de energía. Especialmente, consideraremos una integración en la arquitectura metro/acceso, donde los segmentos metro y de acceso son consolidados en un solo dominio y donde las tecnologías de acceso fijas y móviles comparten la misma infraestructura [6] Este trabajo esta organizado de la siguiente manera: en la Sección II proporcionamos una visión general de los trabajos relacionados, mientras en la Sección III describimos nuestra 
referencia integrada a la arquitectura de red. En la Sección IV se describe los modelos de potencia adoptados para el equipo de red, y en la Sección V definimos nuestras estrategias para la conmutación coordinada on y off de los MSes para ahorrar energía. Finalmente, en la Sección VI mostramos ejemplos numéricos ilustrativos basados en simulaciones para cuantificar la ganancia en nuestro enfoque. La Sección VII concluye este trabajo.

\section{TRABAJOS RELACIONADOS}

Existen algunos estudios que hacen frente al consumo de energía y a la evaluación de la eficiencia energética relacionada con el despliegue de un sistema distribuido de servidores VoD cercano a los usuarios. Ref. [5] fue el primer trabajo en investigar la existencia de una compensación entre la energía que se necesita para el transporte y la energía necesaria para el almacenamiento de contenido de videos. Ref. [7] define un Integer Linear Program (ILP) para caches ubicadas mas cerca de los usuarios mientras se minimiza la energía de consumo total. Ambos trabajos asumen que los contenidos son replicados cerca de los usuarios, pero los servidores todavía se colocan solo en la red core, mientras que nuestro trabajo considera colocar los contenidos incluso mas cerca de los usuarios (es decir, en la red metro/acceso) para descargar el trafico de la red core. Ref. [8] propone un ILP para la distribución de contenido eficiente enérgicamente: en este caso, todos los nodos del backbone disponen de algunas capacidades de almacenamiento caché y el modelo identifica la solución con mayor efíciencia energética, que permite ahorrar energía apagando algunos enlaces y algunos caches. Incluso en este trabajo (como en Ref. [5] y en Ref. [7]) se refiere a la distribución de contenido en la red, es muy relevante en nuestro trabajo, ya que se ocupa de la estrategia de apagar algunos equipos de la red.

En Ref. [3] se definen cinco lugares estratégicos para la ubicación de contenido $\mathrm{VoD}$ que abarca desde la red de acceso hasta la red core y comparativamente analiza la variación de energía de consumo del transporte y del almacenamiento mientras se almacenan los contenidos en esas ubicaciones. Los autores también definieron algunos umbrales para la tasa de llegada de petición de $\mathrm{VoD}$ que son útiles para entender cuando es eficientemente energético conmutar los servidores de contenido a on/off para las diferentes ubicaciones, considerando un modelo de energía proporcional [9] para todos los equipos de la red. En nuestro enfoque por primera vez analizamos la distribución de contenido en una especifica integración en la arquitectura de red metro/acceso proporcionando una estrategia coordinada de on/off, que puede ser aplicada en un escenario de red dinámica. También superamos la asunción de un modelo de consumo de energía totalmente proporcionada, que consideraremos que es inexacta en la mayoría de los equipos de red de hoy, donde el consumo de energía es solo ligeramente dependiente por la carga transportada [10], como señalaremos en la Sección IV.

\section{LA ARQUITECTURA DE RED}

La Figura 1 muestra la arquitectura integrada metro/acceso considerada en este trabajo, que consiste en una infraestructura de red con topología árbol consolidando los segmentos de metro y de acceso en la red. Todos los nodos

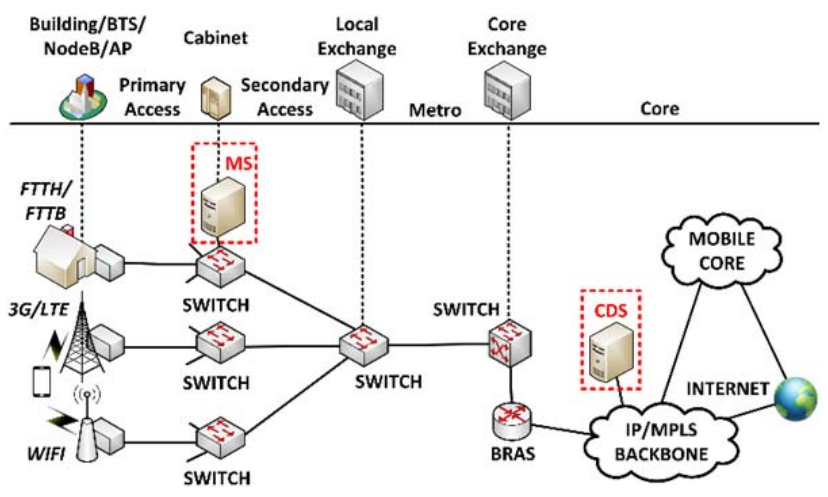

Figura 1. Arquitecura de red integrada metro/acceso con uso compartido de infrasestructura fija y movil.

están activos, es decir, los switches, en contraposición de los dispositivos pasivos como los splitters usados en la redes Ópticas Pasivas (PONs). Para una comparación entre las arquitecturas activas y pasivas, se remite al lector a [11].

Suponemos que un Servidor de Entrega de Contenido (CDS) almacena todo los contenidos es colocado en la red core, entonces añadimos la posibilidad de ubicar los Servidores Metro (MSes) en diferentes posiciones en la red metro/acceso. La arquitectura de red permite compartir recursos entre tecnologías de acceso fijo (p.ej., FTTH, FTTB, ADSL o WiFi) y tecnologías de acceso móvil (p.ej., 3G o LTE). El trafico fijo y móvil es entregado desde/hacia la red core a través de los switches ubicados en la red de topología de árbol metro/acceso. En esta arquitectura los MSes permiten usuarios heterogéneos para recuperar contenido VoD desde locaciones cercanas, permitiendo descarga de trafico en la red core y reduciendo latencia para la recuperación de contenido. Tenga en cuenta la consolidación metro y de acceso así como el uso compartido de infraestructura entre el despliegue de acceso fijo y móvil de las redes de la próxima generación de costos y eficiencia energética [6].

Es importante destacar que la Figura 1 es solo un ejemplo de la arquitectura de referencia, en el que metro/acceso es un árbol de dos etapas y los MSes son colocados en cabinas. Dependiendo de la estructura geográfica y jerárquica de la actual red, la topología de árbol puede variar el numero de etapas y/o de posición de los MSes, e evolucionar hacia una malla mas compleja o topología de anillo. El principal contribución conceptual de este trabajo puede ser fácilmente generalizado también para estos casos. En el recuento del trabajo siempre nos referimos a esta arquitectura de red basada en árbol para la evaluación del consumo de potencia de los servicios VoD.

\section{MODELOS DE POTENCIA DE CONMUTACION Y SERVIDOR METRO}

En esta Sección describimos los modelos de consumo de energía para los equipos de red usados en el despliegue de servicios VoD. Los dos elementos principales, de acuerdo a la arquitectura de red representada en la Sección III, son los switches, que contribuyen al consumo de energía de transporte, y los Metro Servers, que contribuyen al consumo de energía de almacenamiento caché. Especialmente en la posibilidad de conmutar "on" solo un numero mínimo en las 


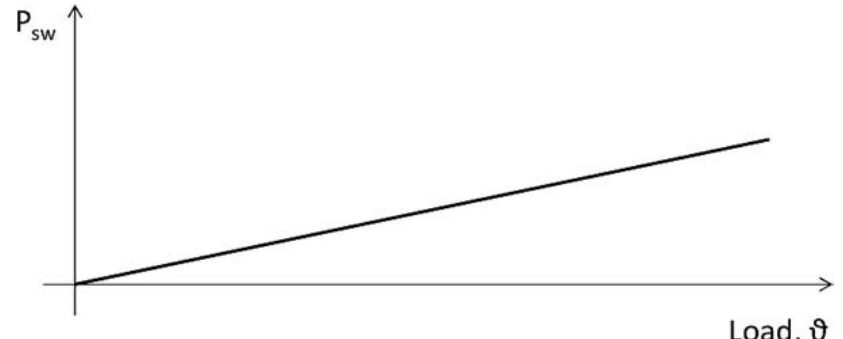

Figura 2a. Modelo de consumo de potencia de conmutación del switch.

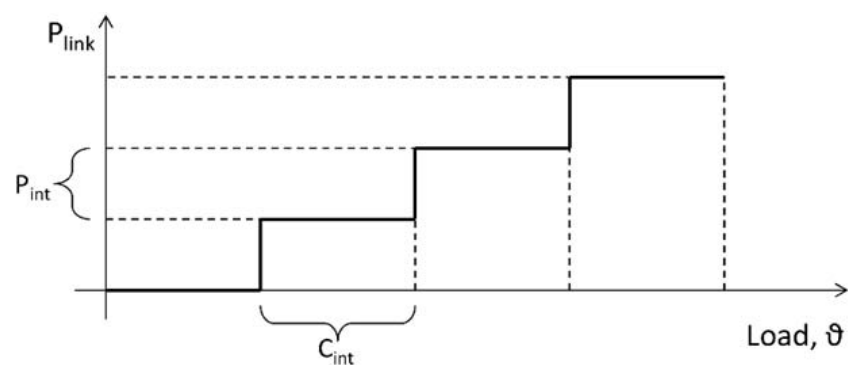

Fig. 2b: Modelo de consumo de potencia de transmisión de un enlace sencillo del switch

interfaces de red para garantizar el servicio VoD proporcionando y ahorrando parte de la energía de consumo por transporte.

\section{A. Modelo del consumo de potencia conmutado}

En nuestro modelo, dividimos el consumo de energía en dos diferentes contribuciones, similares a [12]:

1) Consumo de potencia de conmutación $P_{s w}$

2) Consumo de potencia de transmisión $P_{t r}$

El consumo de potencia de conmutación $P_{S w}$ esta relacionado con todas las operaciones necesarias para reenviar el trafico a partir de una interfaz entrante hasta una interfaz de salida. Por ejemplo, el consumo de potencia del backplane/switching tejidos del switch se considera una contribución en este consumo de potencia. Esta contribución depende altamente del trafico (Fig. 2a).

El consumo de potencia de transmisión $P_{t r}$ esta relacionado con los dispositivos de transmisión (es decir, interfaces de red) que están activos y transmiten datos. En este caso, una vez la interfaz de red esta activa, consume una cantidad fija de trafico transmitido por las interface. Tenga en cuenta que cuando el trafico crece puede ser necesario encender mas de una interface de red (cada una con capacidad de $C_{\text {int }}$ ) sobre los enlaces para servir todas las peticiones de VoD (de esta manera podemos incrementar el ancho de banda $C_{\text {link }}$ de un enlace y evitar el bloqueo de las solicitudes de video). Por el contrario, cuando el trafico disminuye, una o mas interfaces pueden ser apagadas. Entonces, por cada enlace, el consumo de potencia $P_{\text {link }}$ puedes ser representado por una función paso de la carda de trafico transmitidos (Fig. 2b).

Tenga en cuenta que los switches están siempre encendidos ya que tienen que soportar otros servicios de Internet y no solo la entrega de VoD. Por lo tanto, una interfaz e red para cada enlace estará siempre activo para proveer conectividad a todos los nodos en la red integrada metro/acceso y si su consumo de potencia no es considerado

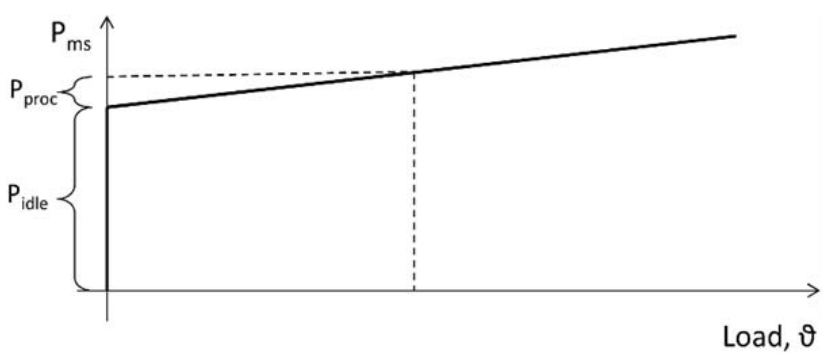

Figura 3. Modelo de consumo de potencia del MS.

en el consumo de potencia de transmisión de $\mathrm{VoD}$ general. Basado en estas observaciones, podemos escribir la formula para $P_{\text {switch }}$, es decir, el consumo de potencia total del para el soporte del servicio $\mathrm{VoD}$ :

$$
\begin{aligned}
& P_{\text {switch }}(\vartheta)=P_{s w}(\vartheta)+P_{t r}(\vartheta)=P_{s w}(\vartheta)+\sum_{i \in V^{+}} P_{\text {link }}^{i}\left(\vartheta_{i}\right) \\
& =P_{s w}(\vartheta)+\sum_{i \in V^{+}}\left(n_{i}\left(\vartheta_{i}\right)-1\right) \cdot P_{\text {int }}
\end{aligned}
$$

donde $\vartheta$ es la carda procesada por el switch expresando en bit/s, $V^{+}$es el conjunto de enlaces salientes, $\vartheta_{i}$ es la carga transmitida sobre el enlace $i \in V^{+}$y $n_{i}\left(\vartheta_{i}\right)$ es el numero de puertos activos para la transmisión sobre el enlace $i$, que depende de la carga de trafico para transmitir sobre el enlace. Llamamos $P_{\text {link }}^{i}$ el consumo de potencia total por el switch para la transmisión sobre el enlace $i$. Tenga en cuenta también que $\vartheta=\sum_{i \in V^{+}} \vartheta_{i}$, porque suponemos que todo el trafico es reenviado y transmitido por el switch, y que la capacidad $C_{\text {link }}^{i}$ para cada enlace $i$ puede ser expresado como $C_{\text {link }}^{i}\left(\vartheta_{i}\right)=$ $n_{i}\left(\vartheta_{i}\right) \cdot C_{\text {int }}$.

Este modelo asume que el switch es capaz de agregar trafico de manera eficiente para minimizar el numero de interfaces de red usadas.

\section{B. Modelo consumo de potencia para el Servidor Metro}

Así como para el modelo de potencia del switch, asumimos que el consumo de potencia de un MS puede ser modelado por diferentes contribuciones:

1) Consumo de potencia idle $P_{\text {idle }}$

2) Consumo de potencia de procesammiento $P_{\text {proc }}$

El idle consumo de potencia $P_{\text {idle }}$ es la carga independiente del consumo de potencia de MS. Como se informo en [10], la cantidad de carga independiente de la energía consumida por el servidor es dominante, es debido principalmente al consumo de potencia idle por las CPUs y por los discos de almacenamiento.

Por el contrario, el consumo de potencia de procesamiento $P_{\text {proc }}$ en la parte de carga dependiente del consumo de potencia de los MS, y es proporcional a la carga proporcionada. Esta contribución es debida principalmente por la carga dependiente del consumo de potencia de los CPUs. Note que el consumo de potencia de los MS hoy esta lejos de ser proporcional, a pesar de las muchas propuestas que han investigado en esta dirección [9]. Por lo tanto, podemos escribir la formula para $P_{m s}$, es decir, el consumo e potencia de el MS (Fig. 3): 
$P_{m s}(\vartheta)=P_{\text {idle }}(\vartheta)+P_{\text {proc }}(\vartheta)$

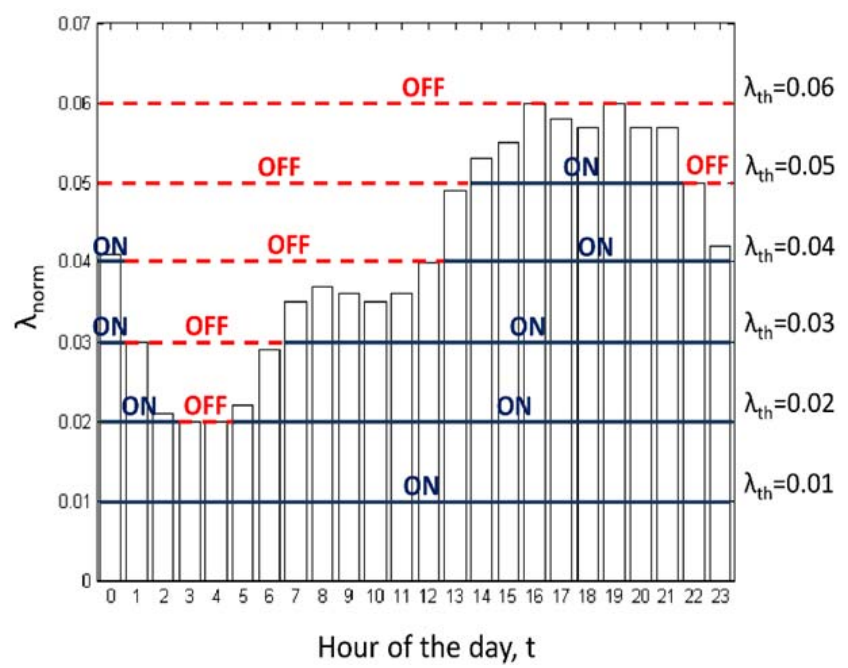

Figura 4. Ejemplo de una distribucion de tasa de solicitud VoD junto a una ilustracion grafica de diferentes patrones on/off obtenidos por la aplicacion de la estrategia on/off para diferentes valores de $\lambda_{\text {th }}$.

En cuanto al CDS, que supone que siempre esta encendido y almacena todos los contenidos como se comentó en la Sección III, consideramos un comportamiento de energía proporcional. Esta suposición sostiene que los centro de datos centralizados de la economía de escala puede ser fácilmente aprovechados para minimizar $P_{\text {idle }}$ de los equipos. Asi, podemos modelar $P_{c d s}$, es decir, el consumo de potencia de el CDS, de la siguiente manera:

$P_{c d s}(\vartheta)=P_{\text {proc }}(\vartheta)$

\section{UNA ESTRATEGIA COORDINADA DE ENERRGIA EFICIENTE PARA CONTROLAR EL ESTADO DE POTENCIA DE LOS MSES DISTRIBUIDOS}

En la Sección IV describimos nuestro modelo de consumo de energía para los switches y los MSes, que rinde dos grandes contribuciones para todo el consumo de energía del servicio VoD: la energía de transporte y la energía de almacenamiento cache. En esta Sección, proponemos hacer frente a la compensación entre esas dos contribuciones de energía cuando se opera los servicios VoD sobre la arquitectura de red descrita en la Sección III. Especialmente, nuestro objetivo es minimizar el consumo de energía conmutando apropiadamente en on o off los MSes durante el día de acuerdo a las condiciones de cambio del trafico. Considerando nuestro modelo de potencia para los switches y los MSes/CDS, la mejor estrategia para encender o apagar los MSes depende de 1) en la normalización la tasa de distribución de solicitudes diurnas del VoD, que definimos como $\lambda_{\text {norm }}(t)$ (es decir, durante el día hay picos de trafico), y 2) en el trafico agregado, $\rho$, que representa el total de throughput del trafico VoD servido para los usuarios finales y el promedio de usuarios en el día, y crece como el numero diario de solicitudes VoD crece. En otras palabras, cuando la red espera ser inundada por un alto numero de solicitudes VoD, será mas conveniente para servirles a los MSes con el fin de ahorrar gran cantidad de energía de transporte. De hecho, en este caso los switches en los niveles superiores de la red metro/acceso y de la red core tendrá que procesar una cantidad inferior de trafico VoD. Así algunas interfaces de red, como se describió en la Sección IV, podría ser apagado, dando lugar a ahorros relevantes en $P_{\text {switch }}$, ya que el trafico es mantenido localmente. Por el contrario, cuando una tasa de solicitud es baja, servir contenido VoD directamente en el CDS puede conducir a ahorrar energía como los MSes pueden apagarse para ahorrar su potencia de consumo $P_{\text {idle }}$.

TABLE I. RESUMEN DE LOS PATRONES ON/OFF OBTENIDOS POR DIFERENTES VALORES DE $\lambda_{t h}$, REPRESENTADOS EN FIG. 4.

\begin{tabular}{|c|c|c|}
\hline & \multicolumn{2}{|c|}{ On/off pattern } \\
\hline$\lambda_{t h}$ & On hours & Off hours \\
\hline 0.01 & All the day & - \\
0.02 & $05: 00 \mathrm{am}-02: 59 \mathrm{am}$ & $03: 00 \mathrm{am}-04: 59 \mathrm{am}$ \\
0.03 & $07: 00 \mathrm{am}-12: 59 \mathrm{am}$ & $01: 00 \mathrm{am}-06: 59 \mathrm{am}$ \\
0.04 & $01: 00 \mathrm{pm}-12: 59 \mathrm{am}$ & $01: 00 \mathrm{am}-12: 59 \mathrm{pm}$ \\
0.05 & $02: 00 \mathrm{pm}-09: 59 \mathrm{pm}$ & $10: 00 \mathrm{pm}-01: 59 \mathrm{pm}$ \\
0.06 & - & All the day \\
\hline
\end{tabular}

En el recuento de esta Sección, primero definimos una estrategia para planificar diferentes periodos on/off (patrones on/off) para los MSes de acuerdo a la tasa de solicitudes diurnas VoD, tratando de capturar diferentes picos de trafico normalizados durante el día. Entonces, definimos cual es el patrón on/off mas eficiente de energía donde un valor especifico para el trafico agregado $\operatorname{VoD} \rho$ es considerado.

Un operador VoD puede planificar diferentes patrones on/off diarios para los MSes mediante el análisis de la tasa de distribución las solicitudes $\mathrm{VoD}$ diurnas, que se supone para poder estimar en un modo preciso por el operador VoD en si. Definiendo el parámetro $\lambda_{t h}$ como el umbral de tasa de solicitudes de $V o D$, podemos establecer la siguiente estrategia on/off.

Definición 1 (Estrategia On/off). Considerando una distribución de tasa de solicitud VoD diurna con el tiempo $\lambda_{\text {norm }}(t)$ y con el umbral de tasa de solicitud $\lambda_{\text {th }}$, los MSes son encendidos o apagados dependiendo de $\lambda_{\text {th }}$ de acuerdo a las siguientes reglas:

- Si $\lambda_{\text {norm }}(t) \leq \lambda_{\text {th }}$, entonces en el tiempo $t$ los MSes son off

- Si $\lambda_{\text {norm }}(t)>\lambda_{\text {th }}$, entonces en el tiempo $t$ los MSes son on

La salida de la estrategia on/off es un patrón diario on/off. Si consideramos diferentes valores para $\lambda_{t h}$, podemos obtener diferentes patrones on/off, cada uno respectivamente con un valor especifico para $\lambda_{t h}$. Para fines ilustrativos, consideremos una distribución de tasa de solicitud VoD diurna estimada en [13] y reportada en la Fig. 4, donde la tasa de 


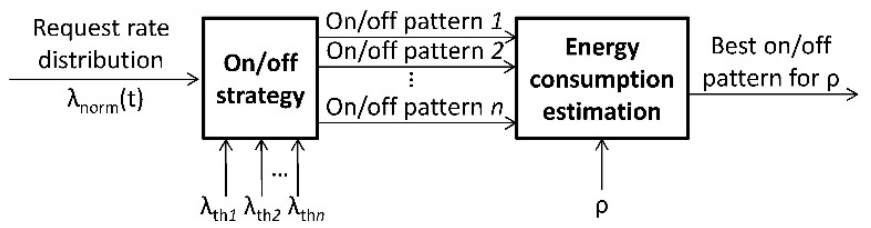

Figura 5. Algortimo Best Energy Pattern (BEPA).

solicitud varia cada hora. En este escenario especifico, aplicamos la estrategia on/off considerando algunos valores para $\lambda_{t h}$, obteniendo el respectivo patrón on/off, como se representa en la Fig. 4 y reportado aun mas en la Table I. Elegimos dichos valores porque estos nos permiten capturar la principal tendencia de la distribución de tasa de solicitud VoD diurna, tenga en cuenta que, en general, entre mas valores de $\lambda_{t h}$ son considerados, mas diferentes patrones on/off pueden ser explorados.

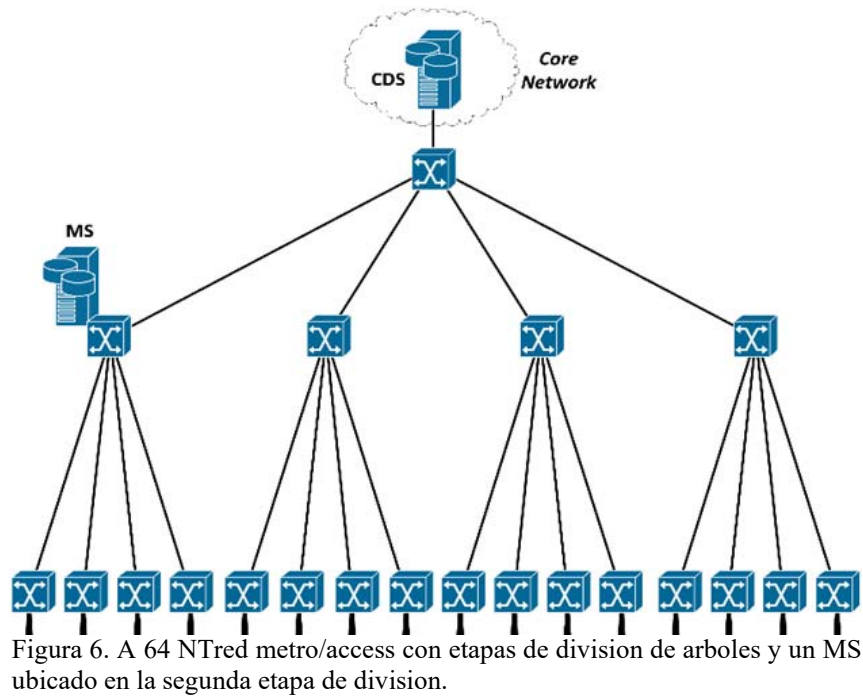

\section{A. Definición del mejor patrón on/off}

Una vez definidos diferentes patrones on/off, el siguiente paso para el operador VoD consiste en explorar y estimar cual es el mejor patrón on/off, es decir, el patrón on/off conduce al minimo consumo de energía diaria, donde el trafico agregado VoD $\rho$ es considerado. Tenga en cuenta que $\rho$ cambia, p.ej. considerando diferentes dias de la semana y/o diferentes periodos del año, el mejor patrón on/off cambia en consecuencia. Nuestro enfoque propuesto permite al operador VoD seleccionar siempre el mejor patrón on/off para cualquier valor de $\rho$, mediante la evaluacion de una estimacion del consumo de enrgia usado en el modelo mostrado en la Sección IV. La Figura 5 muestra toda nuestra estrategia, que llamamos Best Energy Pattern Algorithm (BEPA), para la definicion del mejor patrón on/off.

En la Sección VI aplicamos nuestro algoritmo Best Energy Pattern para simular trafico VoD agregado teniendo en cuenta el consumo de energía del trafico $\mathrm{VoD}$ agregado en los equipos de red de hoy.
TABLE II. SIMULACION DE PARAMETROS.

\begin{tabular}{|c|c|}
\hline Parameter & Value [3][10] \\
\hline $\mathrm{P}_{\mathrm{sw}}$ & $3.31 \cdot 10^{-9} \frac{\mathrm{W}}{\mathrm{bit} / \mathrm{s}}$ \\
$\mathrm{P}_{\text {port }}$ & $15 \mathrm{~W}$ \\
$\mathrm{P}_{\text {proc }}$ & $2.16 \cdot 10^{-8} \frac{\mathrm{W}}{\mathrm{bit} / \mathrm{s}}$ \\
$\mathrm{P}_{\text {idle }}$ & $156 \mathrm{~W}$ \\
$\mathrm{C}_{\text {port }}$ & $1 \mathrm{Gbit} / \mathrm{s}$ \\
Salto promedio de la red core & $10 \mathrm{hops}$ \\
\hline
\end{tabular}

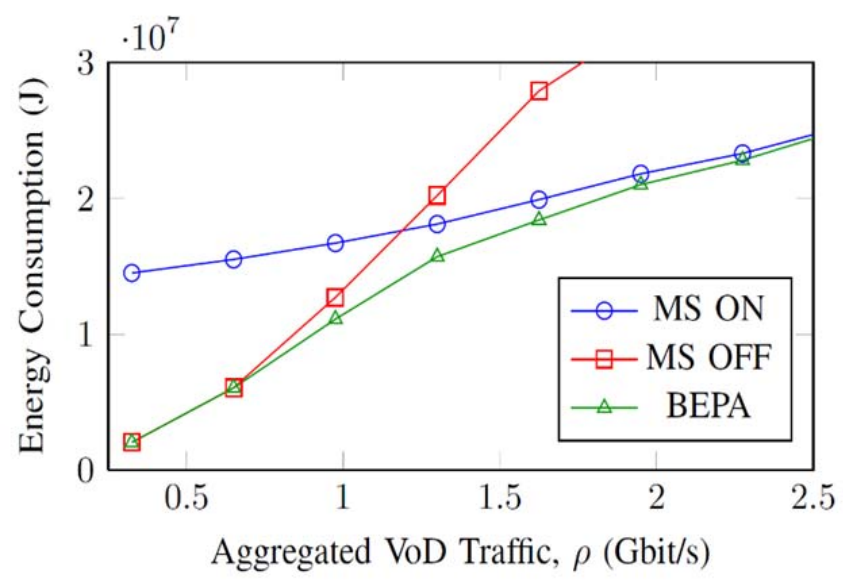

Figura 7. Promedio diario del consumo de energia para los diferentes valores de $\rho$ cuando el MS esta siempre encendido, siempre apagado y la aplicacion del Algortmo the Best Energy Pattern.

\section{RESULTADOS NUMERICOS}

\section{A. Descripcion general del simulador}

Hemos desarrollado un simulador de eventos discretos usando Matlab. Una descripción detallada de nuestro modelo adoptado del contenido $\mathrm{VoD}$ y trafico puede ser encontrado en [11]. En resumen, la distribución de probabilidad de solicitudes de video pueden ser modelado por una distribución Poisson, como en [3], donde cada hora del día la tasa de solicitud es obtenida por una distribución de tasa de solicitud VoD diurna como se muestra en la Fig. 4. Nuestra simulación reproduce, para cada solicitud de video, el requerido ancho de banda, su duración y el asignamiento de un MS o a un CDS. Consideramos el escenario de red en la Fig. 6: simulamos una topología activa de árbol metro/acceso, como se describió en la Sección III, con una relación de división por etapas de 1:4 y tres etapas de división, dando a lugar a una relación global de división de 1:64 (64 terminaciones de red, 64 NT). Como se muestra, consideramos la ubicación de un solo MS en una segunda división de la red. Asumimos que todos los contenidos de video son replicados en el MS y son codificados considerando cuatro diferentes streaming tasas de bit. Los parámetros que utilizamos en nuestra simulación son definidos en [3][10] y son re portados en la Table II.

\section{B. Ganancia del Algoritmo Best Energy Pattern}

La Figura 7 muestra el consumo de energía diario de un servicio $\mathrm{VoD}$ en función del trafico $\mathrm{VoD}$ agregado $\rho$, considerando 1) el MS siempre esta activado (Curva MS ON), 


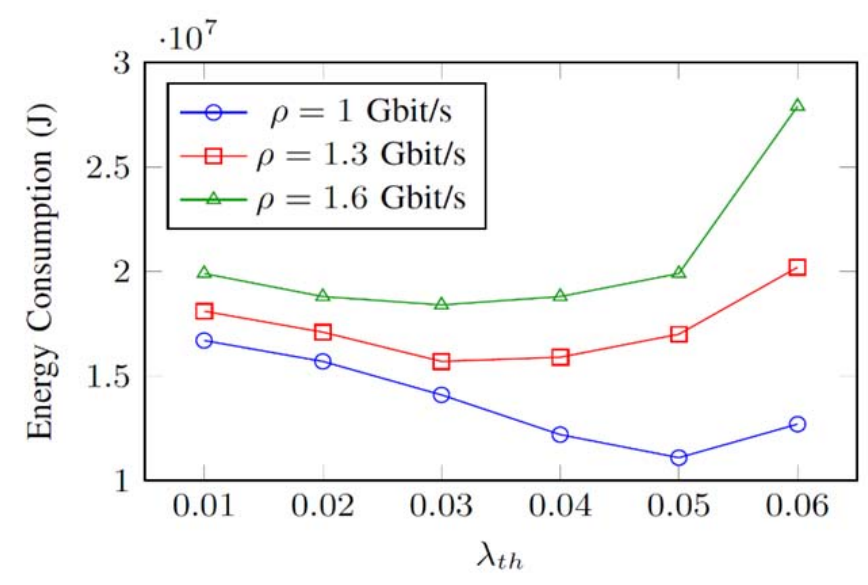

Figura 8. Promedio diario del consumo de energia para los diferentes valores de $\lambda_{\text {th }}$ considerando diferentes valores de $\rho$. La correspondencia entre el respectivo patrón on/off se presentan en la Table I y en la Fig. 4.

2) el MS siempre esta desactivado (Curva MS OFF) y 3) el mejor patrón on/off elige cada calor de $\rho$ aplicando el Algoritmo Best Energy Pattern (Curva BEPA). La Figura 7 muestra que cuando $\rho<1 \mathrm{Gbit} / \mathrm{s}$, las curva del BEPA y del MS ON se sobreponen, es decir, las condiciones de trafico bajo siempre son mas eficientes para servir contenido VoD desde el CDS y desactivando el MS, ya que el consumo total de energía por almacenamiento cache mientras se mantiene el MS siempre activado es mucho mas alto que la energía total de transporte, mientras que el MS siempre esta desactivado. Inversamente, cuando $\rho>1.6 \mathrm{Gbit} / \mathrm{s}$, las curvas del BEPA y del MS OFF se sobreponen, ya que iempre es mas eficiente energéticamente para mantener encendido el MS por todo el día y servir las solicitudes de VoD mas cerca de los usuarios. En este caso, el consumo total de energía de transporte mientras se mantiene siempre desactivado el MS es mucho mas grande que el consumo total de energía por almacenamiento cache mientras se mantiene siempre activo el MS.

Cuando consideramos valores de $\rho$ que conduce a un balance entre el consumo de energía por almacenamiento cache y el consumo de energía de transporte, el Algoritmo Best Energy Pattern permite ahorrar energía conmutando el MS on/off apropiadamente durante el día. Esto sucede alrededor del punto de intersección entre la curva MS ON y la curva MS OFF. En esta configuración, sucede cuando $\rho \simeq 1.3$ Gbit/s. Tenga en cuenta que la intersección entre MS ON y MS OFF se mueve hacia valores mas grandes de $\rho$ cuando el consumo total de energía se convierte menos relevante que el consumo de energía por almacenamiento cache (por ejemplo, si $P_{s w}$ y/o $P_{\text {port }}$ disminuye). Viceversa, la intersección podría pasar para valores bajo de $\rho$.

En la Figura 8 se muestra el consumo de energía diaria para los diferentes valores de $\lambda_{t h}$, mientras se mantenga el trafico VoD agregado $\rho$ constante, alrededor del valor $\rho \simeq 1.3 \mathrm{Gbit} / \mathrm{s}$. Cuando $\rho=1.3 \mathrm{Gbit} / \mathrm{s}$, podemos ver que $\lambda_{t h}=0.03$ conduce a un mínimo consumo de energía. De acuerdo a la coincidencia entre $\lambda_{t h} \mathrm{y}$ el patron on/off reportado en la Table I esto significa que desactivando el MS desde 01:00 am hasta 06:59 am, bajo nuestras asunciones, es la mejor solución, que conduce a un ahorro de energía del $15 \%$ con respecto a tener siempre encendido el MS $\left(\lambda_{t h}=0.01\right)$ y aproximadamente
$25 \%$ con respecto a tenerlo siempre apagado $\left(\lambda_{t h}=0.06\right)$. Considerando $\rho=1 \mathrm{Gbit} / \mathrm{s}$, el ahorro máximo de energía es obtenida para $\lambda_{t h}=0.05$, es decir, cuando el MS esta activada desde 02:00 pm hasta 09:59 pm. En este caso, el patrón on/off permite ahorrar alrededor del $15 \%$ de energía en comparación con mantener siempre apagado el MS y alrededor del $35 \%$ en comparación con mantenerlo siempre encendido. Entonces, en el caso de $\rho=1.6 \mathrm{Gbit} / \mathrm{s}$, podemos ver que la mejor solución es de nuevo tener apagado el MS desde 01:00 am hasta 06:59 am $\left(\lambda_{t h}=0.03\right)$. Considerando la intensidad de trafico, el ahorro de energía es alrededor del $10 \%$ que puede alcanzarse con respecto a tener siempre encendido el MS y $25 \%$ con respecto a mantenerlo siempre apagado.

\section{CONCLUSION}

En este trabajo exploramos el consumo de energía para la entrega del servicio VoD cuando los servidores de video (MSes) son desplegados en una red integrada metro/acceso soportando servicios fijos y móviles. Definimos el modelo de consumo de potencia para los equipos involucrados en la entrada del contendido de video, es decir, los switches, los MSes y el centralizado Content Delivery Server. Nuestro modelo capturo el comportamiento del consumo de energía de la carga dependiente y la carga independiente de los equipos de la red y teniendo en cuenta la posibilidad de conmutar on/off algunas interfaces de red de acuerdo a la intensidad de trafico llevada en los enlaces. Entonces investigamos una estrategia efectiva para definir diariamente un patrón on/off para los MSes dependiendo la variación de la tasa de solicitud VoD durante el día y proporcionamos un herramienta para siempre elegir el mejor patrón on/off dependiendo de los requerimientos de trafico. Investigamos por medio de simulaciones la efectividad de nuestro enfoque considerando el consumo de potencia real en los equipos.

Varios problemas aun permanecen abiertas para futuras investigaciones. Por ejemplo, una estrategia on/off mas compleja para los MSes pueden ser exploradas así como modelos de potencia mas refinados para los equipos. Por otra parte, las solicitudes reales de datos pueden ser usados para mejorar los resultados de la simulación. Actualmente, también estamos investigando las ventajas del paradigma Software Defined Networking (SDN) puede estimular desde la perspectiva de la eficiencia energética, incluyendo una arquitectura centralizada metro/acceso y conmutadores habilitados SDN.

\section{RECONOCIMIENTO}

La investigación que lleva a estos resultados ha recibido financiación de la European Community Seventh Framework Programme FP7/2013-2015 bajo acuerdo de subvención no. 317762 COMBO project.

\section{REFERENCIAS}

[1] "Cisco visual networking index: Global mobile data traffic forecast update, 2012-2017," February 2013.

[2] K. Hinton, J. Baliga, M. Feng, R. Ayre, and R. Tucker, "Power consumption and energy efficiency in the internet," Network, IEEE, vol. 25, no. 2, pp. 6-12, March 2011. 
[3] C. Jayasundara, A. Nirmalathas, E. Wong, and C. A. Chan, "Improving energy efficiency of video on demand services," Optical Communications and Networking, IEEE/OSA Journal of, vol. 3, no. 11, pp. 870-880, November 2011.

[4] E. Di Pascale, D. Payne, and M. Ruffini, "Bandwidth and energy savings of locality-aware P2P content distribution in next-generation PONs," in Optical Network Design and Modeling (ONDM), 2012 16th International Conference on, 2012, pp. 1-6.

[5] J. Baliga, R. Ayre, K. Hinton, and R. Tucker, "Architectures for energyefficient IPTV networks," in Optical Fiber Communication - incudes post deadline papers, 2009. OFC 2009. Conference on, March 2009, pp. $1-3$.

[6] "European FP7 project COMBO," http://www.ict-combo.eu, 2013-2015.

[7] U. Mandal, C. Lange, A. Gladisch, P. Chowdhury, and B. Mukherjee, "Energy-efficient content distribution over telecom network infrastructure," in Transparent Optical Networks (ICTON), 2011 13th International Conference on, June 2011, pp. 1-4.

[8] J. Araujo, F. Giroire, Y. Liu, R. Modrzejewski, and J. Moulierac, "Energy efficient content distribution," in Communications (ICC), 2013 IEEE International Conference on, June 2013, pp. 4233-4238.

[9] L. Barroso and U. Holzle, "The case for energy-proportional computing," Computer, vol. 40, no. 12, pp. 33-37, Dec 2007.

[10] D. Tsirogiannis, S. Harizopoulos, and M. A. Shah, "Analyzing the energy efficiency of a database server," in Proceedings of the 2010 ACM SIGMOD International Conference on Management of Data, ser. SIGMOD '10. plus $0.5 \mathrm{em}$ minus $0.4 \mathrm{emNew}$ York, NY, USA: ACM, 2010, pp. 231-242.

[11] R. Fratini, M. Savi, G. Verticale, and M. Tornatore, "Using replicated video servers for $\mathrm{VoD}$ traffic offloading in integrated Metro/Access networks," in IEEE ICC 2014 - Optical Networks and Systems (ICC'14 ONS), Sydney, Australia, Jun. 2014.

[12] P. Mahadevan, P. Sharma, S. Banerjee, and P. Ranganathan, "A power benchmarking framework for network devices," in NETWORKING 2009. plus 0.5em minus 0.4emSpringer, 2009, pp. 795-808.

[13] W. Tang, Y. Fu, L. Cherkasova, and A. Vahdat, "Medisyn: A synthetic streaming media service workload generator," in Proceedings of the 13th International Workshop on Network and Operating Systems Support for Digital Audio and Video, ser. NOSSDAV '03. plus $0.5 \mathrm{em}$ minus $0.4 \mathrm{em}$ New York, NY, USA: ACM, 2003, pp. 12-21.

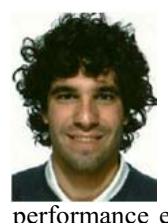

Marco Savi is a PhD student in the Department of Electronic, Information and Bioengineering (DEIB) at the Politecnico di Milano, Italy. He received his B.Sc (2010) and M.Sc (summa cum laude, 2012) in Telecommunication Engineering from Politecnico di Milano. His research interests mainly focus on the security and privacy issues in Smart Grids, on the caching in the access network, and on the Network Functions Virtualization paradigm.

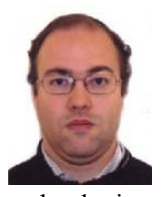

Giacomo Verticale is assistant professor at Politecnico di Milano, Italy. He obtained his $\mathrm{PhD}$ in Telecommunication Engineering in year 2003 from Politecnico di Milano defending a thesis on the performance of packet transmission in UMTS. In the years 1999-2001 he was with the research center CEFRIEL, working on the Voice-over-IP and ADSL technologies. He was involved in several European research projects advancing the Internet technology. His current interests focus on the security issues of the Smart Grid and on Network Functions Virtualization.

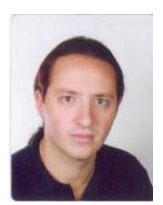

Prof. Massimo Tornatore is an associate professor at Politecnico di Milano, Italy, and an adjunct associate professor at the University of California, Davis, USA. He is author of about 200 technical papers (with 6 best paper awards) and his research interests include the design and engineering of telecom and cloud networks, through optimization and simulation. Prof. Tornatore received a PhD degree in 2006 from Politecnico di Milano.

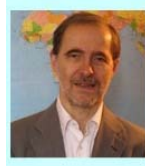

Prof. Achille Pattavina received the Dr. Eng. degree in Electronic Engineering from University La Sapienza of Rome (Italy) in 1977. He has been with Politecnico di Milano in Italy since 1995 as a Full Professor of Communication Networks. He has been author of more than 200 papers and two books in the area of communications switching and networking. His current research interests are in the areas of green ICT, optical networks, switching theory, traffic modeling and broadband convergent access/metro networks. 